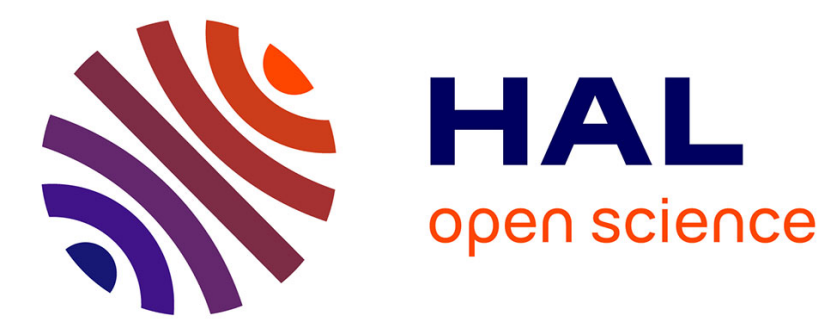

\title{
Chaotic laser voltage: An electronic entropy source
}

Michael J Wishon, Nianqiang Li, D. Choi, D. S Citrin, A. Locquet

\section{To cite this version:}

Michael J Wishon, Nianqiang Li, D. Choi, D. S Citrin, A. Locquet. Chaotic laser voltage: An electronic entropy source. Applied Physics Letters, 2018, 112 (26), pp.261101. 10.1063/1.5025433. hal-03007209

\section{HAL Id: hal-03007209 \\ https://hal.science/hal-03007209}

Submitted on 16 Nov 2020

HAL is a multi-disciplinary open access archive for the deposit and dissemination of scientific research documents, whether they are published or not. The documents may come from teaching and research institutions in France or abroad, or from public or private research centers.
L'archive ouverte pluridisciplinaire HAL, est destinée au dépôt et à la diffusion de documents scientifiques de niveau recherche, publiés ou non, émanant des établissements d'enseignement et de recherche français ou étrangers, des laboratoires publics ou privés. 


\title{
Chaotic Voltage: An Electronic Entropy Source
}

\author{
Michael J. Wishon, ${ }^{1,2}$ Nianqiang Li ${ }^{3}$ D. Choi, ${ }^{1,2}$ D.S. Citrin, ${ }^{1,2}$ and Alexandre Locquet ${ }^{1,2, *}$ \\ ${ }^{1}$ UMI-2958 Georgia Tech-CNRS, Georgia Tech Lorraine, 2 rue Marconi, 57070 Metz, France \\ ${ }^{2}$ School of Electrical and Computer Engineering, Georgia Institute of Technology, \\ 77 Atlantic Drive NW, Atlanta, Georgia 30332-0250 \\ ${ }^{3}$ School of Computer Science and Electronic Engineering, \\ University of Essex, Wivenhoe Park, Colchester CO4 3SQ, UK
}

(Dated: February 9, 2018)

\begin{abstract}
The chaotic terminal voltage dynamics of a semiconductor laser subjected to external optical feedback are utilized to directly generate electronic random number streams with minimal postprocessing at rates of $40-120 \mathrm{~Gb} / \mathrm{s}$, thus obviating the need for optical-to-electrical conversion and facilitating integration with high-speed computers and devices. Further, a comparison of the terminal voltage to the optical intensity being utilized as entropy sources is performed. It is shown that the voltage dynamics have an inherently larger entropy, a reduction in delay signature, and a more suitable distribution for generating random bit streams.
\end{abstract}

Random bit sequences are commonly utilized in cryptography, machine learning, and simulations. But, due to the need for fast rates and ease of generation most random bit sequences are generated using deterministic algorithms and not truly random entropy sources. Therefore, fast physical random bit generators that can be easily integrated into computers or electronic systems are of interest for these applications. In this paper, we point the way to such a device by utilizing the chaotic terminal voltage of a laser diode. Specifically, laser diodes undergo a profound modification to their dynamical variables, i.e., carrier density, photon number, and optical phase, when subjected to optical self-feedback. It is wellknown that these systems can display chaotic dynamics and have been utilized as an archetypical testbed for nonlinear dynamics [1] and for high-speed information processing applications [2]. In the latter context, the first use of sampled chaotic optical intensity fluctuations as an entropy source utilized for high-speed random number generation was demonstrated by Uchida et al. [3]. The generated bit streams were an order of magnitude faster than any other previously utilized physical source. Since then, various realizations and improvements utilizing optical chaos have been demonstrated allowing for faster generation rates up to $\mathrm{Tb} / \mathrm{s}$. Various methods based on creating symmetric distributions without bias through post-processing techniques have been proposed, for example, using derivatives [4-6] and finite differences [7]. To further improve the paradigm, other novel chaotic laser sources and schemes have been demonstrated, such as, through bandwidth enhancement [8], in photonic integrated circuits [9], ring lasers [10, 11], heterodyning [12], terahertz optical asymmetric demultiplexers [13], chaotic solitary vertical-cavity surface-emitting lasers [14], polarization rotated feedback [15], as well as, long on-chip optical feedback [16]. Additionally, fast rates have been demonstrated utilizing parallel significant bits [17] and

* alocquet@georgiatech-metz.fr parallel-laser schemes [18]. The bit streams in most of the previously cited works are post-processed offline. Interestingly in some cases, real-time implementations have been demonstrated, using delayed feedback lasers [19] and photonic integrated circuits [20]. Recently, an interesting concept, physical white chaos, has been proposed and the enhanced chaos leads to random number generation at $320 \mathrm{~Gb} / \mathrm{s}$ [21]. While there have been many improvements and schemes demonstrated in the field of random number generators based on chaotic lasers, in almost all cases advanced post-processing, complex experimental schemes, optical to electronic conversion, and/or exotic components are required for passing the standard randomness tests and for achieving ultrafast bit rates.

In this Letter, we demonstrate that the chaotic laser terminal voltage $V(t)$, as a surrogate for carrier density $n(t)$ in the gain medium [22-25], can be used as a novel, fully electronic, entropy source for random number generation at rates of 40-120 GS/s, where only simple postprocessing is required when compared to utilizing the optical intensity $I(t)$. In order to understand the reason behind this improvement, we compare the voltage entropy source with the commonly utilized optical intensity which was measured simultaneously from the same chaotic laser. Specifically, the entropy sources are compared utilizing distributions, delay-correlation, and permutation entropy analysis. It is shown that the simple physical measurement of $V(t)$ is an inherently superior entropy source compared to the optical intensity, $I(t)$. Further, our approach has the benefit of simplicity, while still generating random bit streams at tens to hundreds of $\mathrm{Gb} / \mathrm{s}$. Specifically, the system, for the first time, offers a fast electronic entropy source that can be used to generate $\mathrm{Gb} / \mathrm{s}$ streams without any optical-to-electrical conversion, thus facilitating the use of this entropy source for high-speed computing.

In the experiment, an unpackaged multi-quantum well DFB laser was utilized $[25,26]$ operating at $1550 \mathrm{~nm}$. The laser was subjected to delayed feedback from a mirror placed at a distance $L$ resulting in a delay time of $\tau$. The sub-nanosecond AC component of $V(t)$ was 


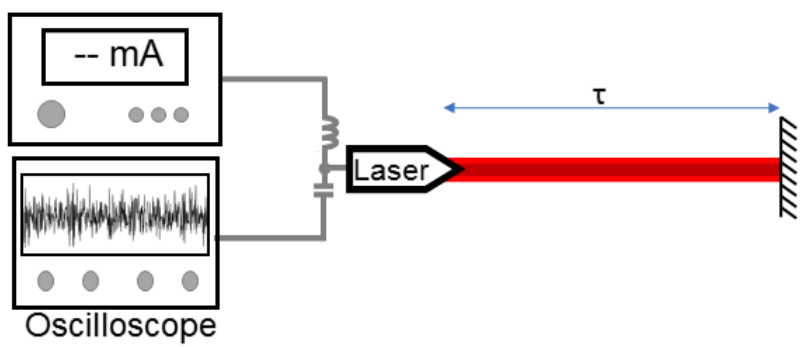

FIG. 1. The relatively simple experimental setup is shown. An unpackaged, MQW laser is subjected to self-feedback from a mirror, after a time delay, $\tau$, and distance $L$. The AC component of the terminal voltage is measured using a realtime oscilliscope (40 GS/s and $12 \mathrm{GHz}$ ).

measured using a high-speed oscilloscope (40 GS/s) connected to a RF probe, bias- $T$, and amplifier, as shown in Fig. 1. The resulting voltage time-series $V(t)$ were converted to binary sequences by simply retaining the least significant bit (LSB) from the output of the 8-bit analog-to-DC converter (ADC). The generated streams were then subjected to the NIST test for randomness [27]. In Table I, we can see that this simple operation can lead to bit stream at $40 \mathrm{GS} / \mathrm{s}(=1 \mathrm{LSB} \times 40 \mathrm{GS} / \mathrm{s})$, that pass the NIST suite. Furthermore, we studied the maximum number of bits that could be extracted and utilized in real-time. We found that the bit sequence generated by extracting 3 LSBs from the ADC with a bitwise exclusive-or operation with a delayed version of the bit sequence can also pass all the randomness tests in the NIST suite. This means that this still relatively simple scheme allows for a $120 \mathrm{~Gb} / \mathrm{s}(=3 \mathrm{LSBs} \times 40 \mathrm{GS} / \mathrm{s})$ realtime bit stream generation with minimal post-processing. The XOR operation is a typical technique used to remove the bias from bit sequences [28]. Of note, the simultaneously measured $I(t)$ does not pass the NIST test with a single LSB with or without the XOR. Further, we wanted to verify that the chaotic dynamics are needed in order to pass the NIST test with 1 LSB. Thus, we tested if amplified spontaneous emission noise in $I(t)$ and $V(t)$ would pass by blocking the feedback and they did not.

The bit streams are able to pass the NIST test without any complicated post-processing due to the nature of the voltage entropy source. In the following, we use different tests to confirm the superiority of the voltage as an entropy source. As shown in our previous work [7, 29], the distribution of $I(t)$ is known to be exponential and skewed which leads to bias. This bias can be removed using post-processing techniques and through complex experimental schemes, as demonstrated by the state-of-theart. But, as we will show, the terminal voltage dynamics have an inherently broader distribution. From Fig. 2, it is clear that the distribution given by $V(t)$ is broader than the exponential distribution of $I(t)$ which leads to significantly less bias in the voltage measurement. It is well known that $n(t)$ follows the optical intensity in the laser cavity as a result of the oscillations between photon and
TABLE I. Results of the NIST test for the terminal voltage measurement using a 1 Gbit stream. For the time series here the cavity length was $30 \mathrm{~cm}$, the injection current was twice threshold, and the feedback strength is chosen such that the laser is operated in coherence collapse [25]. For the tests that produce multiple $\mathrm{P}$-values and proportions the worst case is shown.

\begin{tabular}{lccc} 
STATISTICAL & P-VALUE PROPORTION & RESULT \\
\hline Frequency & 0.6798 & 0.992 & SUCCESS \\
Block frequency & 0.6392 & 0.992 & SUCCESS \\
Cumulative sums & 0.7095 & 0.988 & SUCCESS \\
Runs & 0.1927 & 0.993 & SUCCESS \\
Longest run & 0.6579 & 0.986 & SUCCESS \\
Rank & 0.4299 & 0.992 & SUCCESS \\
FFT & 0.4578 & 0.988 & SUCCESS \\
Nonperiodic template & 0.0156 & 0.983 & SUCCESS \\
Overlapping template & 0.3994 & 0.985 & SUCCESS \\
Universal & 0.1421 & 0.990 & SUCCESS \\
Approximate entropy & 0.3094 & 0.988 & SUCCESS \\
Random excursions & 0.5070 & 0.986 & SUCCESS \\
Random variant & 0.3917 & 0.993 & SUCCESS \\
Serial & 0.5281 & 0.994 & SUCCESS \\
Linear complexity & 0.4376 & 0.985 & SUCCESS \\
\hline
\end{tabular}

electron populations. This leads to the expectation that the same distribution would be obtained from both measurements. In fact, neither the magnitude of the optical field nor $n(t)$ are measured directly. Instead, we measure $I(t)=|E(t)|^{2}$ and $V(t) \propto \ln [n(t)]$, as shown in [23], with $E(t)$ the complex electric field. An explanation for the difference in the distributions can be explained through a change of variable extension of the best fit exponential distribution of $I(t)$ and a natural $\log$ relationship of $n(t)$ to the measured $V(t)$. Specifically, if we let $I(t)$ be a continuous random variable with probability density function $f_{I}(I)$, and let $V(t)=u(I)$ be an invertible function of $I$ with inverse function $I=g(V)$, then the probability distribution of $V$ is $f_{V}(V)=f_{I}[g(V)]\left|g^{\prime}(V)\right|$. If $f_{I}(I)$ is exponential [29]

$$
f_{I}(I)=\frac{1}{b} \exp \left(-\frac{|x-\mu|}{b}\right)
$$

then through change of variable we obtain

$$
f_{V}(V)=\frac{1}{b} \exp \left(-\frac{|\exp [k V(t)]-\mu|}{b}\right) \exp [k V(t)]
$$

with $\mu$ the mean, and $k$ and $b$ distribution fitting parameters.

Figure 2(b), shows the experimental fit to $f_{I}(I)$, and Fig. 2(a) demonstrates that Eq. 2 is a good fit of the experimental distribution from the voltage, $V(t)$. The goodness of fit was checked using the KolmogorovSmirnov test and underlying distributions matched Eqs. 1 and 2 with a $2 \%$ and $6 \%$ confidence, respectively. This observation confirms that the shape of the observed distribution of $v(t)$ is due to the inherent, physical, log relation between the carrier density and the voltage. Small 

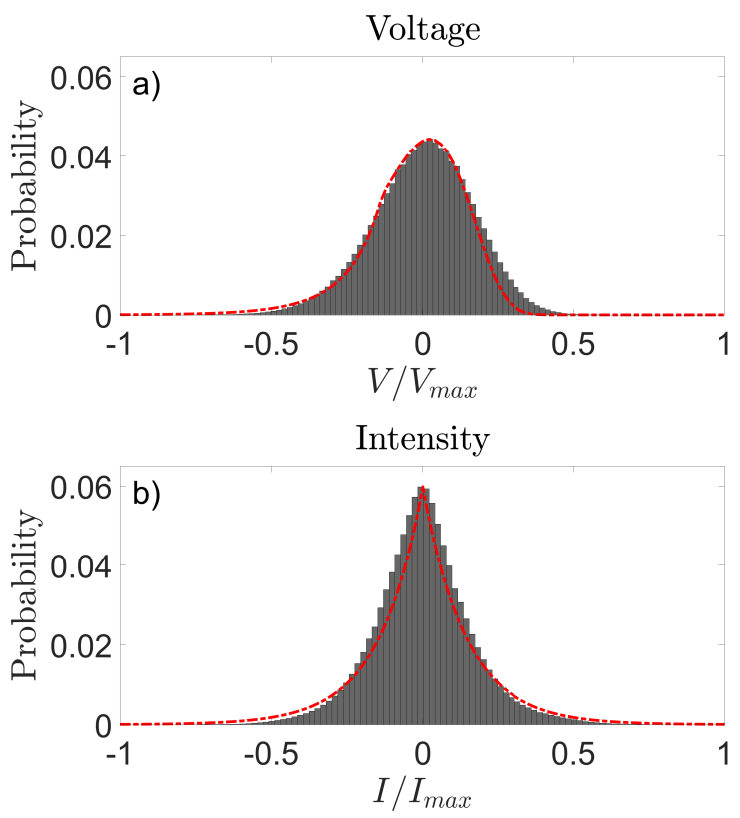

FIG. 2. Comparison of $V(t)$ and $I(t)$ distributions from experimental data. The intensity is fit with the exponential distribution [29] and the voltage is fit with the distribution from Eq. 1. Both distributions were checked using the KolmogorovSmirnov test, and were found to match the underlying distributions with a confidence of $6 \%$ (a) and $2 \%$ (b), respectively. The voltage is broader in nature, thus, it is better suited for generating bit streams. The spreading of the distribution results from the fact the voltage is measured, which is the natural $\log$ of the carriers [23].

deviations in the fit of $v(t)$ might arise from experimental error, noise in the amplifier, as well as, propagating error in the original fit of $I(t)$. Nonetheless, the measured $V(t)$ leads to a distribution that has an order of magnitude larger variance and is broader resulting from the natural $\log$ relationship between $V(t)$ and $n(t)$. The increased symmetry leads to a decrease in bias of the bit sequence [28], thus explaining the possiblility for the LSB of $v(t)$ to pass the NIST test without an additional XOR operation. The increased dispersion or variance means that the distribution is closer to an uniform distribution, leading to a better randomness source. Therefore, the exponential relationship between $n(t)$ and $v(t)$ can be considered as an inherent, physical post-processing that makes the voltage time series more amenable to RNG when compared to $I(t)$.

The second-order statistics of the randomness source also plays a role in its effectiveness. Specifically, reduction of time-delay signatures in the autocorrelation is of importance for generating effective random bit streams. The time-delay signature results from the external-cavity free-spectral range $(1 / \tau)$ or the boundary condition imposed on the system by the external mirror, as shown in Fig. 1. Figure 3 represents the measured auto-covariance function. The result depicted in this Figure demonstrates that there is less correlation $(\sim 75 \%)$ in $V(t)$ than in

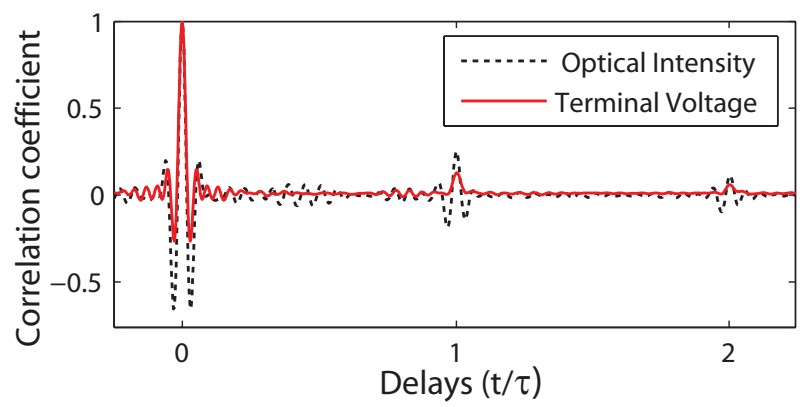

FIG. 3. Comparison of time-delay signature in $I(t)$ and $V(t)$ chaos. There is a reduction in correlation at the delay for $V(t)$. Dashed line: $I(t)$; solid line: $V(t)$.

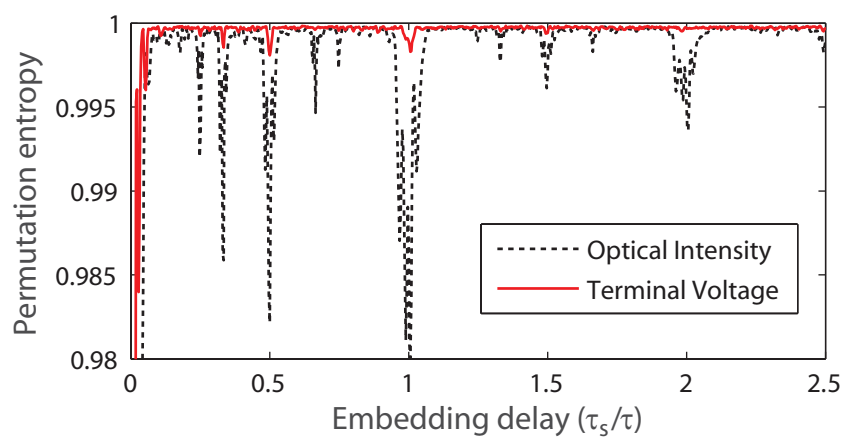

FIG. 4. Permutation entropy estimated with an ordinal pattern length $D=5$ as a function of the embedding delay $\tau_{s} / \tau$. $V(t)$. Dashed line: $I(t)$; solid line: $V(t)$

$I(t)$ at $\tau$, which further illustrates that the voltage entropy source is better suited for generating streams. More broadly, any application that requires the strong suppression or even absence of time-delay signatures [30], for example, chaos-based physical-layer secure communication [31] and chaotic lidar [32], would likely benefit from this chaotic source.

The entropy rate is a crucial parameter that determines the potential and the limits of a chaotic process for RNG $[20,33,34]$. To gain more insight into the advantages of using $V(t)$ over the commonly used $I(t)$, we carry out a permutation-entropy analysis. This analysis is relatively simple to compute and provides convincing information on the complexity even when computed on experimental data $[16,35-38]$. It has been widely used for quantifying dynamical complexity in chaotic systems since the seminal work of Bandt and Pompe [38]. In particular, it has been shown that permutation entropy evaluated at specific timescales for external-cavity lasers can recover important features characterized by KolmogorovSinai entropy rate, and is consistent with the notion of weak and strong chaos $[35,36]$. Here we compare the results of permutation entropy estimated from the two physical entropy sources, i.e., $I(t)$ and $V(t)$ in chaotic lasers. Of importance, the entropy calculations were per- 
formed on the raw data extracted by the ADC without any post-processing, as suggested in [33]. Therefore, the physical entropy rate from the two different sources is compared, and deterministic post-processing is removed from the entropy calculation [33].

For the computation of permutation entropy, we use the ordinal pattern length $D=5$ and assume the embedding delay $\tau_{s}$ as the control parameter, in order to obtain a measure of the dynamical complexity over multiple timescales. The results are shown in Fig. 4, clearly indicating that $V(t)$ has larger values of permutation entropy than $I(t)$ computed at the embedding delay $\tau_{s}$ matching harmonics and subharmonics of delay time $\tau$. Accordingly, the entropy estimation in conjunction with results in Figs. 2 and 3 further confirm that the proposed terminal voltage $V(t)$ is a promising physical entropy source for fast random number generation, and thus providing a physical explanation of the main results in this work.

Further, the results are robust to experimental conditions if the dynamics are chaotic (coherence collapse). Specifically, $L$ was varied from $30-60 \mathrm{~cm}$, the injection current from 1.5-2.5 times threshold, and the feedback within $10 \%$ and the bit streams generated from the measured chaotic voltage time series passed the NIST test. Contrarily, those extracted from the simultaneously measured chaotic optical intensity and spontaneous emission noise time series did not pass the NIST test with the same post-processing.

In conclusion, we have demonstrated that the simple terminal voltage measurement of a laser subjected to optical feedback from a mirror can be utilized to generate random bit streams at $40-120 \mathrm{~Gb} / \mathrm{s}$ thus circumventing the need for optical-to-electrical conversion. Such an electronic entropy source is therefore envisaged to be more easily integrated with high-speed computers. Our results demonstrate that the chaotic terminal voltage is a superior entropy source when compared to optical chaos, benefiting from a broader distribution, a smaller delay signature, and a larger permutation entropy at the delay. More importantly, the scheme demonstrated here is significantly simpler to implement by only requiring a laser, mirror, $\mathrm{ADC}$, and minimal or no post-processing; depending on the speed of the ADC, it is possible to generate random numbers at tens of $\mathrm{Gb} / \mathrm{s}$ while remaining robust to variations in various experimental parameters. Also, it represents a direct electronic source of chaos that is being utilized for generating these bit streams without optical to electrical conversion. Thus, the system could be implemented as a discrete package into an electronic application that directly needs random number streams at very fast rates. Moreover, any application which requires a reduction in time-delay signature and larger entropy, such as chaos-based secure communications and chaotic lidar, could also benefit from these voltage dynamics.

We gratefully acknowledge the financial support of the Conseil Regional de Grand Est, and the Fonds European de Developement Regional (FEDER).
[1] Junji Ohtsubo, "Semiconductor lasers: stability, instability and chaos," $\mathbf{1 1 1}$ (2012).

[2] Miguel C Soriano, Jordi García-Ojalvo, Claudio R Mirasso, and Ingo Fischer, "Complex photonics: Dynamics and applications of delay-coupled semiconductors lasers," Rev. Mod. Phys. 85, 421 (2013).

[3] Atsushi Uchida, Kazuya Amano, Masaki Inoue, Kunihito Hirano, Sunao Naito, Hiroyuki Someya, Isao Oowada, Takayuki Kurashige, Masaru Shiki, Shigeru Yoshimori, et al., "Fast physical random bit generation with chaotic semiconductor lasers," Nat. Photonics 2, 728-732 (2008).

[4] I Reidler, Yaara Aviad, M Rosenbluh, and Ido Kanter, "Ultrahigh-speed random number generation based on a chaotic semiconductor laser," Phys. Rev. Lett. 103, 024102 (2009).

[5] Ido Kanter, Yaara Aviad, Igor Reidler, Elad Cohen, and Michael Rosenbluh, "An optical ultrafast random bit generator," Nat. Photonics 4, 58-61 (2010).

[6] Jianzhong Zhang, Yuncai Wang, Ming Liu, Lugang Xue, $\mathrm{Pu} \mathrm{Li}$, Anbang Wang, and Mingjiang Zhang, "A robust random number generator based on differential comparison of chaotic laser signals," Opt. Express 20, 7496-7506 (2012).

[7] Nianqiang Li, Byungchil Kim, VN Chizhevsky, A Locquet, M Bloch, DS Citrin, and Wei Pan, "Two approaches for ultrafast random bit generation based on the chaotic dynamics of a semiconductor laser," Opt. Express 22, 6634-6646 (2014).
[8] Kunihito Hirano, Taiki Yamazaki, Shinichiro Morikatsu, Haruka Okumura, Hiroki Aida, Atsushi Uchida, Shigeru Yoshimori, Kazuyuki Yoshimura, Takahisa Harayama, and Peter Davis, "Fast random bit generation with bandwidth-enhanced chaos in semiconductor lasers," Opt. Express 18, 5512-5524 (2010).

[9] Apostolos Argyris, Stavros Deligiannidis, Evangelos Pikasis, Adonis Bogris, and Dimitris Syvridis, "Implementation of $140 \mathrm{gb} / \mathrm{s}$ true random bit generator based on a chaotic photonic integrated circuit," Opt. Express 18, 18763-18768 (2010).

[10] Romain Modeste Nguimdo, Guy Verschaffelt, Jan Danckaert, Xaveer Leijtens, Jeroen Bolk, and Guy Van der Sande, "Fast random bits generation based on a single chaotic semiconductor ring laser," Opt. Express 20, 28603-28613 (2012).

[11] T Butler, C Durkan, D Goulding, S Slepneva, B Kelleher, SP Hegarty, and G Huyet, "Optical ultrafast random number generation at $1 \mathrm{tb} / \mathrm{s}$ using a turbulent semiconductor ring cavity laser," Opt. Lett. 41, 388-391 (2016).

[12] Xiao-Zhou Li and Sze-Chun Chan, "Heterodyne random bit generation using an optically injected semiconductor laser in chaos," IEEE J. Quantum. Electron. 49, 829-838 (2013).

[13] Pu Li, Yuanyuan Sun, Xianglian Liu, Xiaogang Yi, Jianguo Zhang, Xiaomin Guo, Yanqiang Guo, and Yuncai Wang, "Fully photonics-based physical random bit generator," Opt. Lett. 41, 3347-3350 (2016). 
[14] Martin Virte, Emeric Mercier, Hugo Thienpont, Krassimir Panajotov, and Marc Sciamanna, "Physical random bit generation from chaotic solitary laser diode," Opt. Express 22, 17271-17280 (2014).

[15] Neus Oliver, Miguel Cornelles Soriano, David W Sukow, and Ingo Fischer, "Fast random bit generation using a chaotic laser: approaching the information theoretic limit," IEEE J. Quantum. Electron. 49, 910-918 (2013).

[16] Guy Verschaffelt, Mulham Khoder, and Guy Van der Sande, "Random number generator based on an integrated laser with on-chip optical feedback," Chaos: An Interdisciplinary Journal of Nonlinear Science 27, 114310 (2017).

[17] Yasuhiro Akizawa, Taiki Yamazaki, Atsushi Uchida, Takahisa Harayama, Satoshi Sunada, Kenichi Arai, Kazuyuki Yoshimura, and Peter Davis, "Fast random number generation with bandwidth-enhanced chaotic semiconductor lasers at $8 X 50 \mathrm{gb} / \mathrm{s}$," IEEE Photonics Technol. Lett. 24, 1042-1044 (2012).

[18] JG Wu, X Tang, ZM Wu, GQ Xia, and GY Feng, "Parallel generation of 10 gbits/s physical random number streams using chaotic semiconductor lasers," Laser Phys. , 1-5 (2012).

[19] Anbang Wang, Pu Li, Jianguo Zhang, Jianzhong Zhang, Lei Li, and Yuncai Wang, "4.5 gbps high-speed realtime physical random bit generator," Opt. Express 21, 20452-20462 (2013).

[20] Kazusa Ugajin, Yuta Terashima, Kento Iwakawa, Atsushi Uchida, Takahisa Harayama, Kazuyuki Yoshimura, and Masanobu Inubushi, "Real-time fast physical random number generator with a photonic integrated circuit," Opt. Express 25, 6511-6523 (2017).

[21] Anbang Wang, Longsheng Wang, $\mathrm{Pu} \mathrm{Li}$, and Yuncai Wang, "Minimal-post-processing 320-gbps true random bit generation using physical white chaos," Opt. Express 25, 3153-3164 (2017).

[22] RF Kazarinov and RA Suris, "Heterodyne reception of light by an injection laser," Zhurnal Eksperimental'noi i Teoreticheskoi Fiziki 66, 1067-1078 (1974).

[23] AA Sahai, B Kim, D Choi, A Locquet, and DS Citrin, "Mapping the nonlinear dynamics of a laser diode via its terminal voltage," Opt. Lett. 39, 5630-5633 (2014).

[24] D Brunner, MC Soriano, X Porte, and I Fischer, "Experimental phase-space tomography of semiconductor laser dynamics," Phys. Rev. Lett. 115, 053901 (2015).

[25] Chien-Yuan Chang, Daeyoung Choi, A Locquet, Michael J Wishon, K Merghem, Abderrahim Ramdane, François Lelarge, A Martinez, and DS Citrin, "A multighz chaotic optoelectronic oscillator based on laser terminal voltage," Appl. Phys. Lett. 108, 191109 (2016).

[26] Chien-Yuan Chang, Michael J Wishon, Daeyoung Choi, Junliang Dong, Kamel Merghem, Abderrahim Ramdane, François Lelarge, Anthony Martinez, Alexandre Locquet, and DS Citrin, "Tunable x-band optoelectronic oscil- lators based on external-cavity semiconductor lasers," IEEE J. Quantum. Electron. 53, 1-6 (2017).

[27] Andrew Rukhin, Juan Soto, James Nechvatal, Miles Smid, and Elaine Barker, Tech. Rep. (Booz-Allen and Hamilton Inc Mclean Va, 2001).

[28] Atsushi Uchida, "Random number generation with chaotic lasers," Optical Communication with Chaotic Lasers: Applications of Nonlinear Dynamics and Synchronization , 445-509.

[29] Nianqiang Li, Byungchil Kim, A Locquet, Daeyoung Choi, Wei Pan, and DS Citrin, "Statistics of the optical intensity of a chaotic external-cavity dfb laser," Opt. Lett. 39, 5949-5952 (2014).

[30] Damien Rontani, Alexandre Locquet, Marc Sciamanna, and DS Citrin, "Loss of time-delay signature in the chaotic output of a semiconductor laser with optical feedback," Opt. Lett. 32, 2960-2962 (2007).

[31] Apostolos Argyris, Dimitris Syvridis, Laurent Larger, Valerio Annovazzi-Lodi, et al., "Chaos-based communications at high bit rates using commercial fibre-optic links," Nature 438, 343 (2005).

[32] Fan-Yi Lin and Jia-Ming Liu, "Chaotic lidar," IEEE journal of selected topics in quantum electronics 10, 991-997 (2004).

[33] Joseph D Hart, Yuta Terashima, Atsushi Uchida, Gerald B Baumgartner, Thomas E Murphy, and Rajarshi Roy, "Recommendations and illustrations for the evaluation of photonic random number generators," APL Photonics 2, 090901 (2017).

[34] Xiao-Zhou Li, Jun-Ping Zhuang, Song-Sui Li, Jian-Bo Gao, and Sze-Chun Chan, "Randomness evaluation for an optically injected chaotic semiconductor laser by attractor reconstruction," Physical Review E 94, 042214 (2016).

[35] Luciano Zunino, Osvaldo A Rosso, and Miguel C Soriano, "Characterizing the hyperchaotic dynamics of a semiconductor laser subject to optical feedback via permutation entropy," IEEE J. Sel. Topics in Quantum Electron. 17, 1250-1257 (2011).

[36] JP Toomey and DM Kane, "Mapping the dynamic complexity of a semiconductor laser with optical feedback using permutation entropy," Opt. Express 22, 1713-1725 (2014).

[37] Nianqiang Li, Luciano Zunino, Alexandre Locquet, Byungchil Kim, Daeyoung Choi, Wei Pan, and David S Citrin, "Multiscale ordinal symbolic analysis of the lang-kobayashi model for external-cavity semiconductor lasers: A test of theory," IEEE J. Quantum. Electron. 51, 1-6 (2015).

[38] Christoph Bandt and Bernd Pompe, "Permutation entropy: a natural complexity measure for time series," Phys. Rev. Lett. 88, 174102 (2002). 